\title{
An Improved Estimation and Gap-Filling Technique for Sea Surface Wind Speeds Using NARX Neural Networks
}

\author{
Murilo T. Silva, ERIC W. GiLl, AND Weimin HuANG \\ Memorial University of Newfoundland, St. John's, Newfoundland and Labrador, Canada
}

(Manuscript received 3 January 2018, in final form 16 March 2018)

\begin{abstract}
This work presents the use of a nonlinear autoregressive neural network to obtain an improved estimate of sea surface winds, taking Placentia Bay, Newfoundland and Labrador, Canada, as a study case. The network inputs and delays were chosen through cross correlation with the target variable. The proposed method was compared with five other wind speed estimation techniques, outperforming them in correlation, precision, accuracy, and bias levels. As an extension, the temporal gap filling of missing wind speed data during a storm has been considered. Data containing a measurement gap from a 40-yr windstorm that hit the same location has been used. The proposed method filled the gaps in the dataset with a high degree of correlation with measurements obtained by surrounding stations. The method presented in this work showed promising results that could be extended to estimate wind speeds in other locations and filling gaps in other datasets.
\end{abstract}

\section{Introduction}

Historical ocean data allow for the study of previous events and testing of mathematical models in the ocean environment. The use of estimation techniques and models on historical data, commonly referred to as hindcasting, is widely used in climate sciences for longterm climate analysis (Suckling et al. 2017), as well as in oceanography for surface wave analysis (Arthur 1951).

Wave estimation methods have greatly evolved in the past 50 years, with different empirical and theoretical models being proposed with increasing complexity, encompassing the evolution in computational processing capabilities. Theoretical models are now in their third generation, relying heavily on numerical techniques to solve the energy transfer equation (Massel 2013). Empirical models, on the other hand, rely on directly measurable data to estimate and forecast waves. Although early empirical models were criticized for being too simplistic (Kinsman 1965), they have advanced to the point in which they would perform as well as theoretical models under simple wind regimes (Dexter 1974). More recently, machine learning techniques (Londhe et al. 2016), genetic algorithms (Gaur and Deo 2008), and data mining techniques (Nikoo and Kerachian 2017) have been used to estimate significant wave height.

Corresponding author: Murilo T. Silva, murilots@mun.ca
While there is a vast array of literature on the estimation of ocean measurements from wind data, methods dedicated to the prediction of wind speed from ocean measurements are less common. The issue of wind estimation usually arises in the context of remote sensing, where wind speed information is not always directly available. Some authors, such as Dexter and Theodoridis (1982), have used hindcasting techniques originally designed to estimate ocean measurements to instead estimate wind speeds; one of them, the modified Sverdrup-Munk-Bretschneider (SMB) equation has been widely used to estimate wind speeds from HF radar data (Huang et al. 2002). Some authors, on the other hand, have tried to find a direct relationship between significant wave height and wind speed without resorting to previous wave estimation techniques. Li et al. (2012) and Tokinaga and Xie (2011) have used a power series regression to estimate wind speeds from significant wave heights, while Zeng et al. (2016) have used a neural network to estimate wind speed using different oceanographic measurements. Among the most recent wind speed estimation methods, Clarizia and Ruf (2017) proposed a Bayesian wind speed estimator using significant wave height information inferred from Global Navigation Satellite System reflectometry (GNSS-R) data, while $\mathrm{Li}$ et al. $(2015,2017)$ proposed the use of support vector machines (SVM) to estimate wind speed from a single-Doppler radar. 
TABLE 1. Missing values of wind speed and significant wave height in NOAA buoys (NDBC 2018).

\begin{tabular}{|c|c|c|c|c|c|c|c|c|c|}
\hline \multirow[b]{3}{*}{ Station } & \multirow{3}{*}{$\frac{\text { Water depth }}{(\mathrm{m})}$} & \multicolumn{8}{|c|}{ Missing values (\%) } \\
\hline & & \multicolumn{2}{|c|}{2014} & \multicolumn{2}{|c|}{2015} & \multicolumn{2}{|c|}{2016} & \multicolumn{2}{|c|}{2017} \\
\hline & & WSPD & WVHT & WSPD & WVHT & WSPD & WVHT & WSPD & WVHT \\
\hline 41002 & 3890 & 20.72 & 1.69 & 0.14 & 0.19 & 0.13 & 0.09 & 0.03 & 0.00 \\
\hline 44009 & 43 & 94.07 & 0.22 & 53.89 & 0.38 & 89.00 & 0.92 & 0.06 & 0.06 \\
\hline 44066 & 78 & 0.06 & 3.32 & 0.07 & 7.24 & 93.76 & 0.00 & 16.18 & 0.01 \\
\hline 46002 & 3413 & 0.01 & 1.90 & 17.88 & 5.33 & 45.88 & 0.02 & 35.84 & 0.19 \\
\hline
\end{tabular}

Wind speed estimation techniques can also be used to fill gaps in datasets (Tokinaga and Xie 2011), allowing the use of some data analysis techniques that require a complete time series in order to function properly, such as spectral analysis and the calibration of learning algorithms. Table 1 shows the percentage of missing values of wind speed and significant wave height from National Oceanic and Atmospheric Administration (NOAA) buoys (NDBC 2018).

The results in Table 1 show that the measurement of significant wave height (WVHT) is more reliable than wind speed (WSPD) measurement during normal operation in these stations. Also, being directly exposed to the weather, some anemometers may malfunction or break during extreme wind events (Howden et al. 2008). This was the case during a 40-yr windstorm that hit the Avalon Peninsula, Newfoundland, Canada, in March 2017, with gusts of up to $180 \mathrm{~km} \mathrm{~h}^{-1}$ (MacEachern 2017; Snoddon 2017). During the storm a buoy located at the mouth of Placentia Bay, Newfoundland and Labrador, Canada, stopped registering wind speeds, leaving gaps in the time series. In cases in which the significant wave height is known and the wind speed is missing, an estimation method can be used to fill the gaps in wind speed measurements.

This work presents an autoregressive neural network with external inputs as a method to estimate wind speeds from significant wave heights. This method estimates wind speeds based on historic significant wave height data with a good degree of accuracy. As a further application of the technique, the filling of temporal gaps in wind speeds is considered, using data collected by a buoy located at the mouth of Placentia Bay during the 40-yr storm of March 2017. Section 2 presents the instruments and datasets used in the present work. In Section 3, the proposed method is presented, with a discussion on the general problem of estimating the wind speed from significant wave height and a discussion on previously devised methods to estimate wind speed; these methods will be later used as benchmarks for the proposed method. Section 4 presents training, testing, and gapfilling results. The test results include both a comparison with the previously presented techniques for accuracy, precision, and bias, and tests for estimated wind speed within data gaps using nearby wind speed measurements. Section 5 presents concluding remarks, with suggestions for further developments of this work.

\section{Instruments and datasets}

The data used for the analysis were collected by SmartAtlantic Alliance (2017) at Placentia Bay. There are three buoys at this location, as can be observed in Fig. 1.

The buoy chosen for this study was the one at the mouth of Placentia Bay, marked as " 2 " in Fig. 1. According to SmartAtlantic Alliance (2017), this is an AXYS 3-m-diameter discus buoy, located at $46^{\circ} 58.8^{\prime} \mathrm{N}$, $54^{\circ} 41.1^{\prime} \mathrm{W}$, where the water depth is $230 \mathrm{~m}$. This buoy is equipped with the following instruments, with distances measured from the ocean surface: an anemometer at $4.2 \mathrm{~m}$; thermometers for atmospheric and sea surface temperatures located at 3.5 and $-0.5 \mathrm{~m}$, respectively; a hygrometer and a barometer located at $3.5 \mathrm{~m}$; and a current meter, and sea surface salinity and conductivity meters placed at $-0.5 \mathrm{~m}$. Wave height and direction are measured by a TRIAXYS sensor unit embedded in the buoy's payload (AXYS Technologies 2017). This sensor unit consists of three accelerometers, three gyroscopes, a fluxgate compass, and a processor, allowing it to retrieve significant wave height, maximum wave height, peak wave period, wave mean direction, and wave mean spread. By definition, significant wave height is the average of the highest third of the wave heights measured in a given period, which can be obtained either directly from height measurements or from the moments of the wave spectrum (Stewart 2008).

The mouth of Placentia buoy is capable of retrieving the following measurements (SmartAtlantic Alliance 2017): average wind speed and direction, peak wind (or gust) speed, air temperature, humidity, dewpoint, barometric pressure, sea surface temperature, maximum wave height, sea surface salinity, sea surface conductivity, significant wave height, peak wave period, wave mean direction, wave mean spread, current speed, and current direction. 


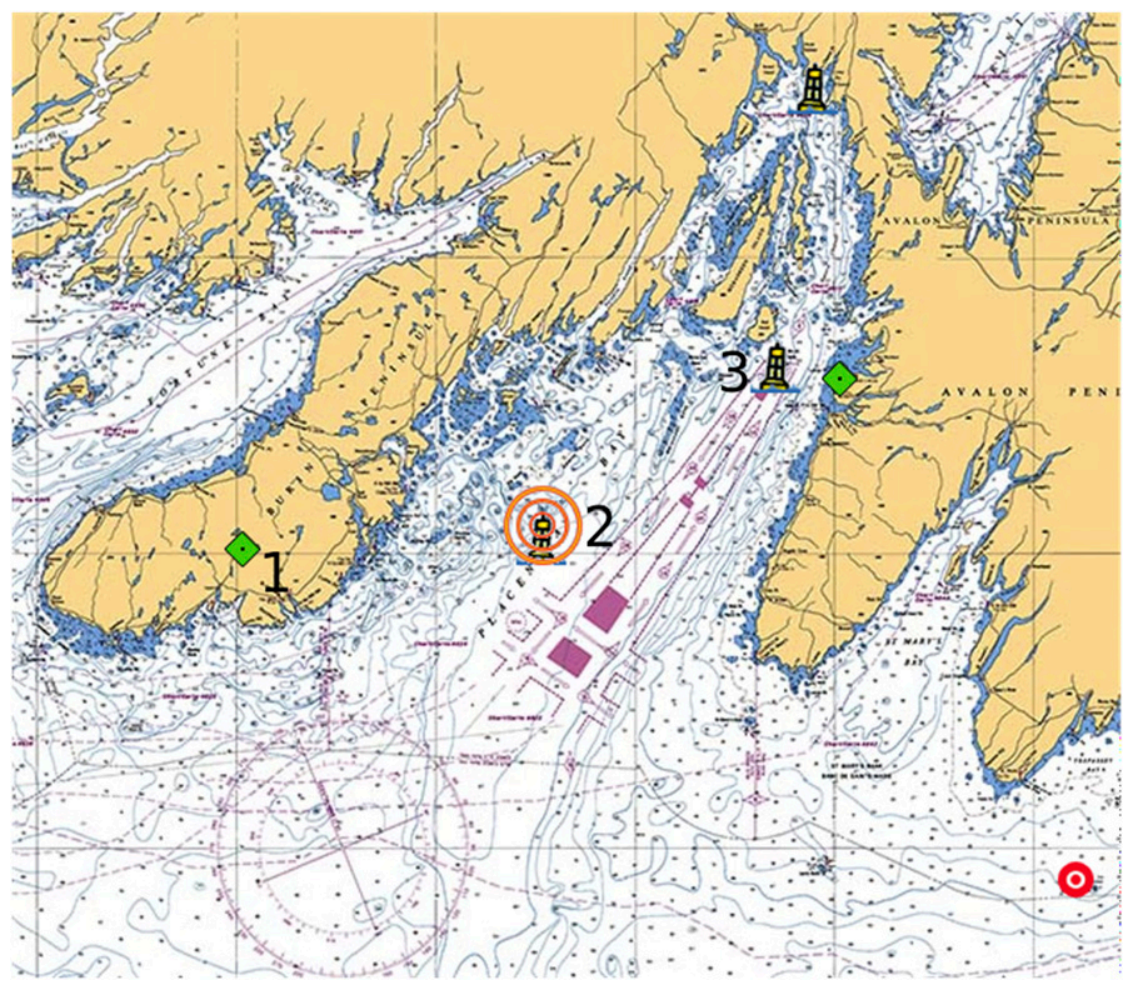

FIG. 1. Location of buoys and weather stations in Placentia Bay: 1-St. Lawrence weather station, 2-mouth of Placentia Bay buoy, and 3-Red Island Shoal buoy. Adapted from SmartAtlantic Alliance (2017).

According to Environment Canada (2015), average wind speed and direction are defined as the 2-min average values ending at the time of observation, with the direction measured from true north. Peak wind speed, or gust speed, is defined as the maximum sudden change in wind speed, registered if the highest peak speed is at least $5 \mathrm{kt}\left(1 \mathrm{kt}=0.51 \mathrm{~m} \mathrm{~s}^{-1}\right)$ higher than the 2-min average wind speed, or if the highest peak is at least $15 \mathrm{kt}$.

Historical data were obtained from this buoy, with measurements between August 2006 and March 2017. From this historical dataset, the two longest continuous data sequences in both wind speed and significant wave height were selected: the longest, between 3 May 2013 and 20 February 2014, was chosen to be the training dataset, while the second longest, between March and December 2009, was used to test the wind estimation methods.

A dataset of the month of March 2017 was used for the gap-filling experiment. During this month there were $29 \mathrm{~h}$ of missing wind speed data for the mouth of Placentia Bay buoy, representing 3.9\% of the total hours. From these, only $1 \mathrm{~h}$ also had missing significant wave height data, meaning that $28 \mathrm{~h}$ of data can be estimated. To evaluate the estimates of wind speed within the gaps, data from two neighboring stations, Red Island
Shoal buoy and St. Lawrence weather station, shown as 1 and 3, respectively, in Fig. 1, were collected.

The Red Island Shoal buoy has the same characteristics as the mouth of Placentia Bay buoy (SmartAtlantic Alliance 2017), while the St. Lawrence weather station is an inland station managed by Environment and Climate Change Canada, with data available online (Environment and Climate Change Canada 2017).

Because of the position of the anemometer, wind speeds measured on both buoys were corrected for wind speeds at $10 \mathrm{~m}$ from the sea surface using the method presented in U.S. Army Corps of Engineers (2012a), while data provided by the inland station was corrected for wind speeds over the sea using the regression model proposed by Powell (1982), combined with the temperature correction proposed by Resio and Vincent (1977) and U.S. Army Corps of Engineers (2012b).

\section{Wind speed estimation}

\section{a. Previous wind speed estimation methods}

The nature of the ocean-atmosphere system allows us to infer that the significant wave height is a good candidate for the input of a wind speed estimation system 
(Kinsman 1965). Although it was conceived as an empirical measurement, the significant wave height was theoretically defined with respect to the ocean wave spectrum after the introduction of spectral analysis to studies of the upper ocean (Hoffman and Karst 1975).

According to Kitaigorodskii (1962), the power spectral density of the ocean surface can be written as a function dependent on the acceleration resulting from gravity, wind friction velocity, and fetch. This definition was later simplified by Pierson and Moskowitz (1963), who substituted the dependence on friction velocity, which cannot be directly measured, by wind speed at a given height $z$ from the ocean surface $U_{z}$. In this case the relationship between wind speed and significant wave height can be written as

$$
H_{s}=F\left(U_{z}\right)=4\left[\int_{0}^{\infty} S\left(\omega \mid U_{z}\right) d \omega\right]^{1 / 2}
$$

where $H_{s}$ is the significant wave height, $S$ is the power spectral density of the ocean surface, and $\omega$ is the radial frequency of the ocean surface. Therefore, in light of the relationship presented in Eq. (1), the problem of wind speed estimation is an inverse one. Mathematically, it can be described as

$$
U_{z}=F^{-1}\left(H_{s}\right) .
$$

A well-known relationship between wind speed and significant wave height is the SMB equation (Bretschneider 1952, 1958). Huang et al. (2002) applied a modification to the SMB equation (Dexter and Theodoridis 1982) to the problem of wind speed estimation from HF radar data, in which the fetch was substituted by the peak wave period to make the equation dependent on only measurable radar parameters. The modified SMB relationship can be written as

$$
\frac{g H_{s}}{U_{10}^{2}}=0.26 \tanh \left[\left(\frac{3.5 g T_{m}}{U_{10}}\right)^{3 / 2} \times 10^{-2}\right],
$$

where $g$ is the acceleration resulting from gravity, $H_{s}$ is the significant wave height, $U_{10}$ is the wind speed measured at $10 \mathrm{~m}$ from sea level, and $T_{m}$ is the peak wave period. If the estimation of significant wave height is desired, then Eq. (3) can be solved analytically; however, if the SMB equation is applied to estimate wind speeds, then it can only be solved numerically.

With the modified SMB equation, Dexter and Theodoridis (1982) have also presented a wind estimation method based on the JONSWAP spectral model (Hasselmann et al. 1973). Mathematically, this method can be written as

$$
U_{10}=9.11 \times 10^{3}\left(\frac{H_{s}^{2}}{g T_{m}^{3}}\right) .
$$

Unlike the SMB equation, the wind speed in the JONSWAP model can be obtained without resorting to numerical solvers. Both expressions in Eqs. (3) and (4) are limited by the fetch $F$, meaning that they are valid only if

$$
F<10^{4} \frac{U_{10}^{2}}{g} \mathrm{~m}
$$

Another method used to estimate wind speed from significant wave height was presented by the U.S. Army Corps of Engineers (2012a) in their Coastal Engineering Manual (CEM). The CEM model can be written as

$$
\frac{g H_{s}}{u_{*}^{2}}=4.13 \times 10^{-2}\left(\frac{g F}{u_{*}^{2}}\right)^{\frac{1}{2}} .
$$

The main difference between the CEM model and all previously presented methods is the fact that it uses friction velocity $u *$ instead of $U_{10}$. Friction velocity and $U_{10}$ are related as follows:

$$
u_{*}^{2}=C_{d} U_{10}^{2},
$$

where $C_{d}$ is the drag coefficient. In the literature there are several formulations for the drag coefficient. However, the expression used in the CEM model (U.S. Army Corps of Engineers 2012a) is

$$
C_{d}=1 \times 10^{-3}\left(1.1+3.5 \times 10^{-2} U_{10}\right) .
$$

Using the ancillary relationship

$$
\frac{g T_{m}}{u_{*}}=0.651\left(\frac{g F}{u_{*}^{2}}\right)^{\frac{1}{3}}
$$

Equation (5) can be rewritten as

$$
\frac{g H_{s}}{u_{*}^{2}}=7.863 \times 10^{-2}\left(\frac{g T_{m}}{u_{*}}\right)^{\frac{3}{2}},
$$

which does not directly depend on the fetch. Substituting Eqs. (6) and (7) into Eq. (8), and after some algebraic manipulations, the $C E M$ model can be expressed in terms of $U_{10}$ as

$$
U_{10}^{3}+31.4286 U_{10}^{2}=7.4751 \times 10^{8}\left(\frac{H_{s}^{4}}{g^{2} T_{m}^{6}}\right),
$$


which is clearly a third-order equation with respect to wind speed. Therefore, the estimated wind speed using the CEM model is the real positive root of Eq. (9).

A different approach from all previously mentioned methods was presented by Tokinaga and Xie (2011). Instead of relying on physical relationships of the air-sea interaction, they have used a power series regression that can be written as

$$
U_{10}=\alpha H_{s}^{\beta}+\gamma,
$$

where $\alpha, \beta$, and $\gamma$ are constants to be solved based on training data.

Since they are universal function approximators (Cybenko 1989; Hornik et al. 1989), artificial neural networks (ANNs) can be used to estimate the inverse function in Eq. (2). A method using ANN to estimate wind speed from oceanographic data was proposed by Zeng et al. (2016), in which a wind speed prediction method for high-frequency (HF) radar data using a backpropagation (BP) neural network was presented. The inputs to this method were wind direction, significant wave height, and average wave period, having wind speed as the output, with no feedback loop. The network was trained first using buoy data and later applied to radar measurements.

\section{b. Proposed method}

From Eq. (1) it is evident that the dataset used to estimate $F^{-1}$ should contain both significant wave height and wind speed information; however, other ancillary data could be included, provided they have sufficient correlation with the wind speed. Also, since the interaction between wind and ocean surface is a dynamic one, a suitable network architecture for this kind of problem is the nonlinear autoregressive artificial neural network with exogenous entries (NARX ANN; Haykin 2009). Therefore, the goal of this work is to estimate wind speed from ocean data by using a NARX neural network.

The structure of a NARX ANN used in this work can be observed from Fig. 2, where $z^{-1}$ are delay operators, and $\mathbf{W}_{n-1, n}$ and $b$ are weight vectors and biases of the network, respectively, with $n$ being the layer to which the weight vector is referred.

The autoregressive characteristic of the neural network is manifested either as a feedback loop, where previous estimates are presented as inputs, or as an open loop, where previous measurements are presented instead. In this work the network will change between these two modes, presenting estimates wherever measured data are missing and presenting previous measurements otherwise. Although the NARX neural network contains both delays and a feedback loop, it operates in the same way as a regular backpropagation neural network (Siegelmann et al. 1997).

One distinguishing characteristic of the proposed method is that, besides the power series and the backpropagation artificcial neural network (BP ANN), all other techniques have a fixed structure. The proposed method and the BP ANN are based on a universal function approximator paradigm that allows for a high level of adaptability. To a lesser extent, the power series model also allows for adaptability, since different values of $\alpha, \beta$, and $\gamma$ will produce a different estimator. The remaining techniques- namely, the SMB, the CEM, and the JONSWAP modelsall have fixed structures, with no room for adaptability.

Although both methods use artificial neural networks, the proposed method is markedly different from the one proposed by Zeng et al. (2016). The main difference between the two is the regressive nature of the proposed method. While the BP ANN uses concurrent measurements to estimate the wind speed, the proposed method exploits the fact that wind speed over the ocean surface has a delayed effect on the highest one-third of the waves for higher sea states, as indicated by the PhillipsMiles mechanism for wave growth and generation (Massel 2013). Therefore, by adjusting the number of delays in an autoregressive neural network, it is possible to cover the maximum correlation between wind speed and significant wave height in both low sea states, where the time gap between acting wind and significant wave height is shorter, and high sea states, where the wind will not have immediate effect on significant wave height. This difference between the proposed method and the BP ANN is shared with the other techniques presented in the previous section: they all rely on concurrently measured variables to estimate wind speed.

\section{Results and discussion}

\section{a. NARX network setting}

Even though significant wave height is a theoretically good candidate for the network input, a cross-correlation analysis was performed to test this assumption and to select other possible network inputs. During this test the cross correlation of each variable with the wind speed is calculated. The variables that have the highest correlation with wind speed were selected. Any variable that was measured through the anemometer was discarded from the input analysis. The cross-correlation analysis was performed on the longest continuous wind speed sequence of the dataset, between 3 May 2013 and 20 February 2014. 


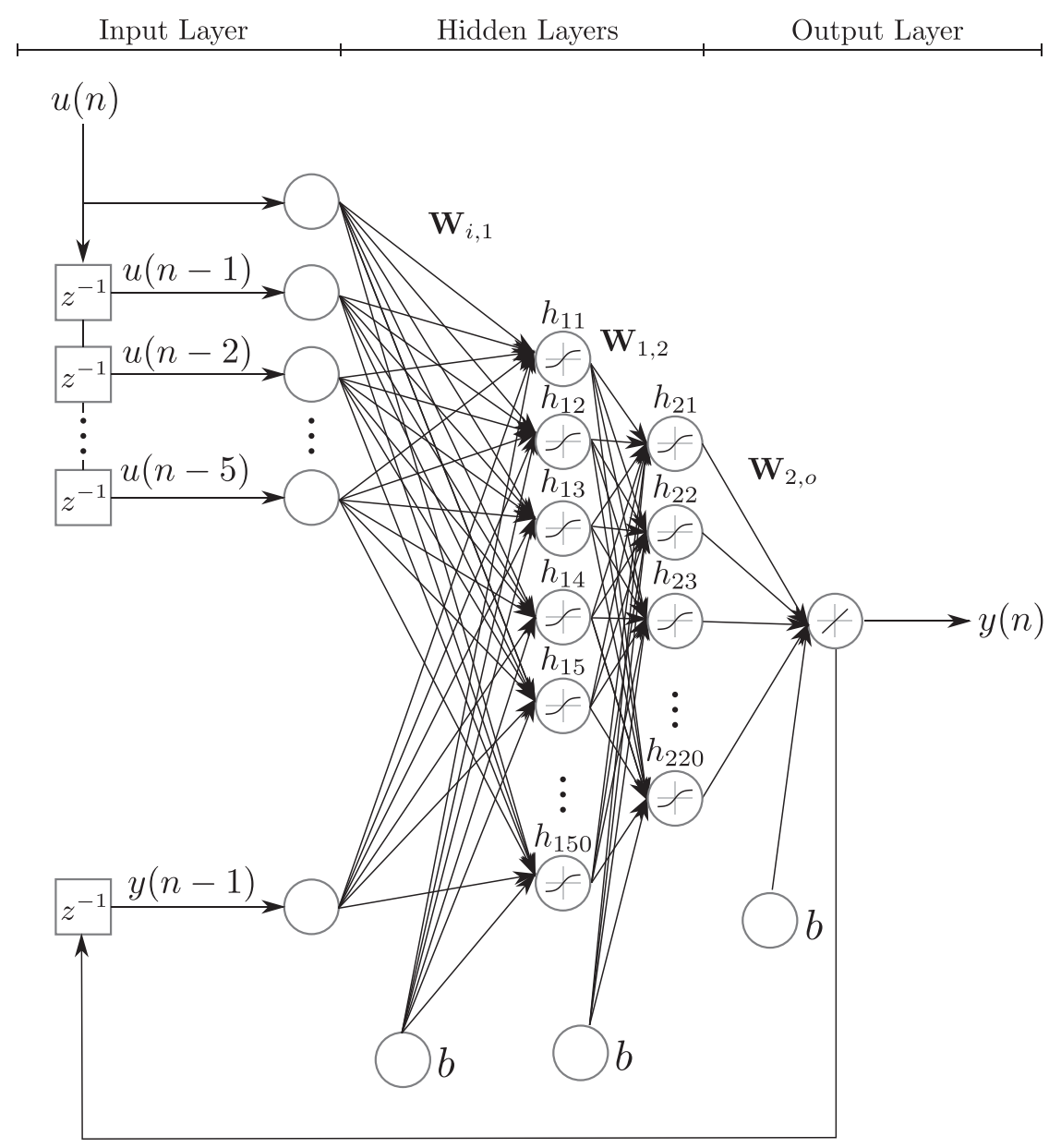

FIG. 2. Scheme of the NARX neural network used in this work.

Figure 3 presents the cross correlation between wind speed and the following variables: wind speed, gust velocity, maximum and significant wave heights, and peak wave period. The remaining variables had either too small cross correlation with the wind speed to appear in the plot or were discontinuous within the observed period.

Analyzing the cross correlations presented in Fig. 3, it is evident that wind speed and gust have the highest cross correlation, followed by maximum wind speed and significant wave height, and peak wave period, respectively. Evidently, both wind speed and gust would have the highest correlation. However, one of them is the desired output and the other is also measured through the anemometer; therefore, they are not eligible input candidates.

The peak wave period presents a peak correlation with the wind speed of $0.31 \mathrm{~m} \mathrm{~s}^{-1}, 17.5 \mathrm{~h}$ after the wind in question has blown over the ocean surface. During this period the wind could have changed speed and direction several times, and considering the dynamics of a wave field, it is not expected that the peak wave period would respond to local winds after this length of time. It is also observed that even after this period, other measurements, such as significant wave height, present a higher correlation than the peak wave period. Therefore, the use of the peak wave period as one of the inputs would greatly increase the complexity of the neural network, and it would add only slightly correlated information to the network. Therefore, the peak wave period will not be considered as one of the inputs of the NARX neural network.

The remaining input candidates are significant and maximum wave heights. Since both variables have the same crosscorrelation behavior within the observation interval, with minimal discrepancies, and the theory presented in the previous sections shows a clear relationship between significant wave height and wind speed, only significant wave height was selected as the input variable of the NARX neural network. Another factor in favor of significant wave height is that it is a measurement that can be obtained in a remote sensing setting, which allows for future applications of the method to radar data. 


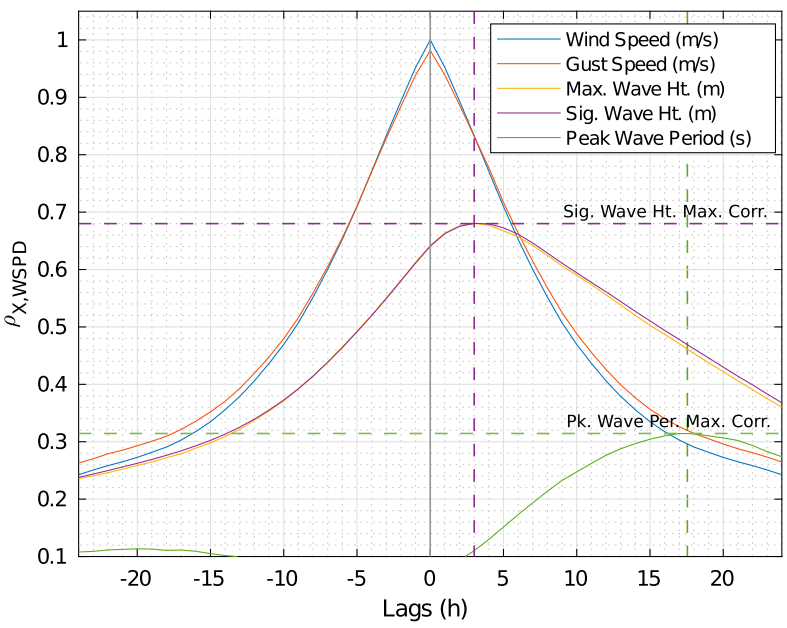

FIG. 3. Cross correlation between wind speed and all other variables in the mouth of Placentia Bay buoy training dataset.

Another important piece of information that can be obtained from Fig. 3 is the fact that the wind at a given time $t$ has a maximum correlation with the significant wave height at $t+3 \mathrm{~h}$. In other words, to make use of the maximum correlation to predict the wind speed at $t$, the significant wave height at future times must be used. Therefore, in order to turn the NARX ANN into an advance network, the time series must be read backward by the neural network. For the network used in the present work, five delays were applied to the input, which includes the maximum correlation point. On the output feedback loop, only a single delay was considered.

The choice of a two-layer neural network is based on the proven suitability to the solution of inverse problems (Sontag 1992; Haykin 2009). The number of neurons for each layer were chosen by trial and error, such that the cross correlation between input and error and the mean-square error (MSE) were minimized. Satisfactory results were obtained from a network structure with two layers, with 50 neurons in the first layer and 20 in the second layer. Given the nature of neural networks, it is possible to obtain satisfactory results with different network structures. Also, different results can be obtained using the presented network topology if different weights are reached after the training process (Haykin 2009).

\section{b. Training}

To improve the generalization (Beale et al. 2017), 10 neural networks were trained for each method, with different starting weights, training, validation, and testing datasets. The network that returned the lowest MSE was chosen to represent the method. The chosen training algorithm for the proposed method was the
TABLE 2. Training statistics for the neural networks in the present work.

\begin{tabular}{lcccc}
\hline \hline \multirow{2}{*}{ Method } & \multicolumn{2}{c}{ MSE } & & \multicolumn{2}{c}{ Convergence } \\
\cline { 2 - 3 } & $\left(\mathrm{m}^{2} \mathrm{~s}^{-2}\right)$ & & Epoch & Time $(\mathrm{s})$ \\
\hline Proposed & 1.0177 & & 5 & 6.626 \\
BP ANN & 5.3497 & & 66 & 0.471 \\
BP ANN (no $\left.\theta_{w}\right)$ & 5.6522 & & 141 & 1.288 \\
\hline
\end{tabular}

Levenberg-Marquardt (Haykin 2009), while the BP ANN uses the Bayesian regularization algorithm to train the network (Zeng et al. 2016). The same dataset used in the correlation analysis was applied to train the neural networks and it was divided in three parts, $70 \%$ for training, $15 \%$ for validation, and $15 \%$ for testing. The independent test dataset, measured between March and December 2009, was not presented to the neural network during the training phase.

Table 2 presents the training statistics for the selected networks for the proposed method, regular BP ANN, and BP ANN without wind direction information.

From the presented results it is evident that the presented method was able to converge to a lower MSE as compared to either of the BP ANNs. However, both BP ANNs have converged in a fraction of the time used by the proposed method. The removal of wind direction as an input for the BP ANN had a small impact on the accuracy of the method, increasing the training MSE by $5.26 \%$.

The power series regression model was trained using the same training dataset as was presented to the neural networks. The final expression, containing the fitted $\alpha$, $\beta$, and $\gamma$, is

$$
U_{10}=4.349 H_{s}^{0.6874}+1.937 .
$$

The fitted values for the coefficients lie within the typical intervals presented by Tokinaga and Xie (2011). With the coefficients in Eq. (11), the power series method achieved a training root-mean-square error of $3.1994 \mathrm{~m} \mathrm{~s}^{-1}$.

Once these methods have been trained, they can be applied to the test dataset and compared with other estimation methods.

\section{c. Performance analysis}

The proposed method was compared with some of the aforementioned wind estimation techniques, namely, the SMB equation, power series regression, the BP ANN, the CEM equation, and the estimator based on the JONSWAP spectral model. Unlike other previously mentioned techniques, these methods do not depend on instrument-specific data, being able to retrieve wind 


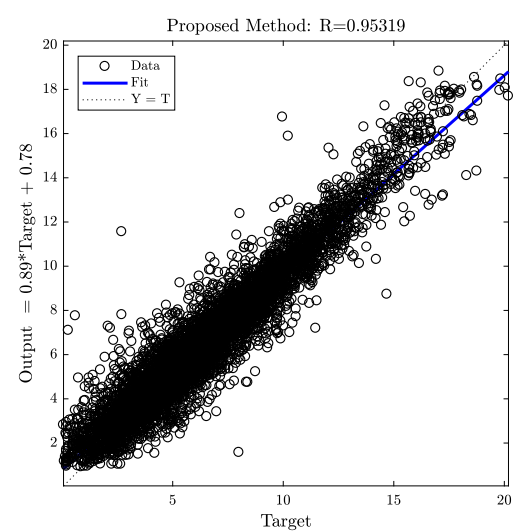

(a)

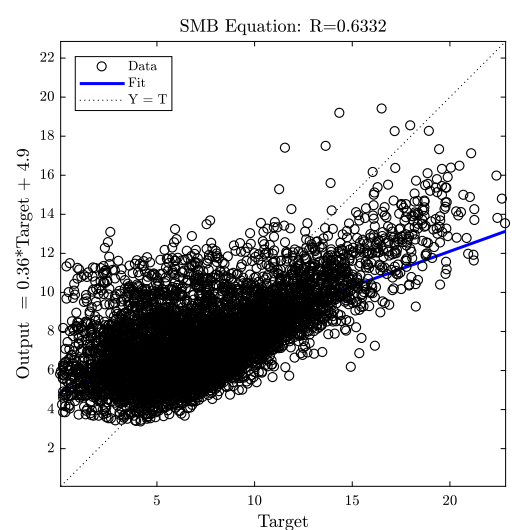

(b)

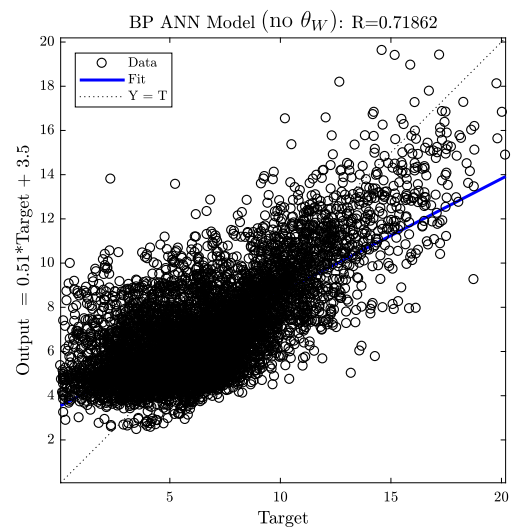

(e)

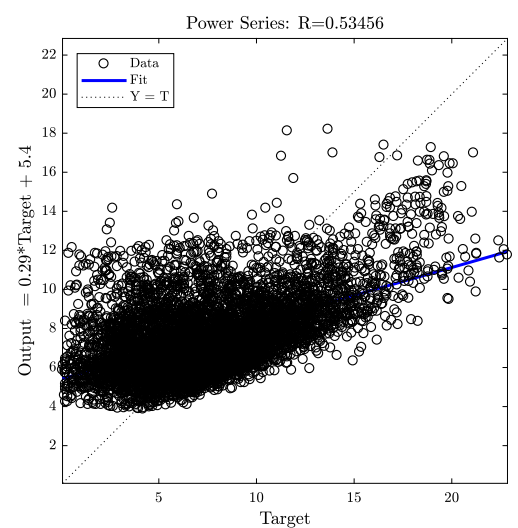

(c)

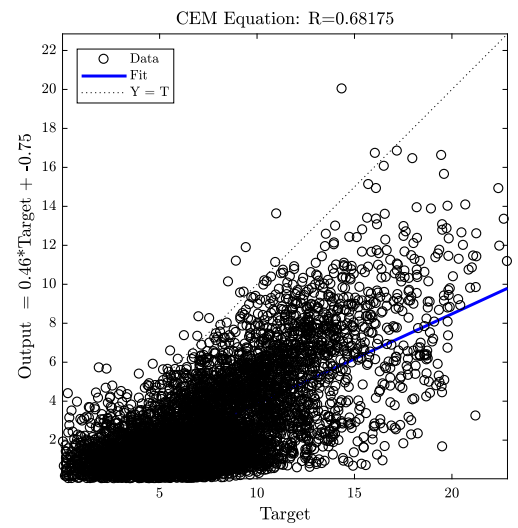

(f)

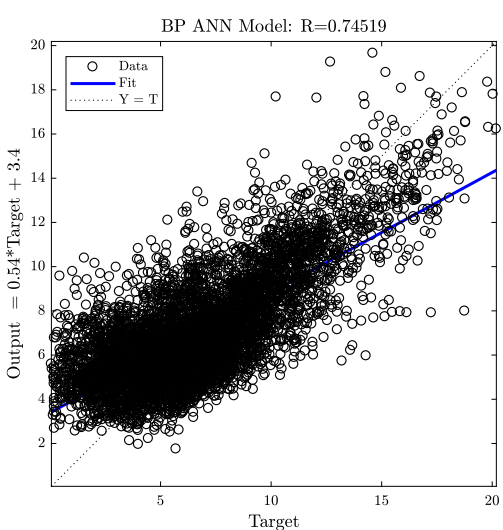

(d)

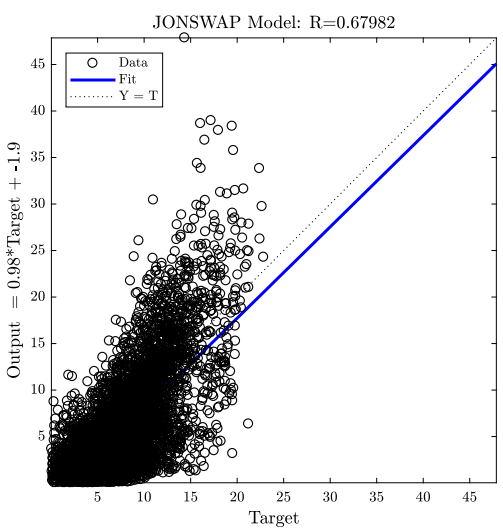

$(\mathrm{g})$

FIG. 4. Correlation analysis between measured and estimated wind speed using the following methods: (a) proposed method, (b) SMB model, (c) power series model, (d) BP ANN model, (e) BP ANN model (no $\theta_{w}$ ), (f) CEM model, and (g) JONSWAP model.

speed estimations solely from other meteorological measurements.

As previously explained, the test dataset was the second-longest continuous sequence for wind speed and significant wave height data, measured between March and December 2009. Figure 4 shows the linear regression between measured and estimated wind speeds for each method. In all cases the correlations between measured and estimated values were statistically significant, having $p$ values smaller than 0.5 .

From the scatterplots in Fig. 4, it is evident that the proposed method, with a correlation coefficient above 0.95 , produces better estimates for the wind speed than all other presented methods. The SMB equation, power 
TABLE 3. Performance comparison between wind estimation methods. RMSE denotes root-mean-square error, NRMSE denotes normalized RMSE, MAE denotes mean absolute error, and error IQR denotes interquartile range.

\begin{tabular}{|c|c|c|c|c|c|c|c|}
\hline \multirow[b]{2}{*}{ Method } & \multicolumn{3}{|c|}{ Accuracy } & \multicolumn{2}{|c|}{ Bias } & \multicolumn{2}{|c|}{ Precision } \\
\hline & $\frac{\text { NRMSE }}{(\%)}$ & $\frac{\text { RMSE }}{\left(\mathrm{m} \mathrm{s}^{-1}\right)}$ & $\frac{\mathrm{MAE}}{\left(\mathrm{m} \mathrm{s}^{-1}\right)}$ & $\frac{\text { Error mean }}{\left(\mathrm{m} \mathrm{s}^{-1}\right)}$ & $\frac{\text { Error median }}{\left(\mathrm{m} \mathrm{s}^{-1}\right)}$ & $\frac{\text { Error std dev }}{\left(\mathrm{m} \mathrm{s}^{-1}\right)}$ & $\frac{\text { Error IQR }}{\left(\mathrm{m} \mathrm{s}^{-1}\right)}$ \\
\hline Proposed & 30.34 & 1.14 & 0.75 & $-6.43 \times 10^{-2}$ & $-2.08 \times 10^{-2}$ & 1.01 & 1.19 \\
\hline SMB equation & 77.68 & 2.94 & 2.35 & $-5.49 \times 10^{-2}$ & 0.28 & 2.94 & 4.02 \\
\hline Power series & 84.51 & 3.2 & 2.55 & $-1.76 \times 10^{-14}$ & 0.23 & 3.2 & 4.36 \\
\hline BP ANN & 67.47 & 2.55 & 1.99 & -0.38 & -0.18 & 2.52 & 3.36 \\
\hline BP ANN $\left(\right.$ no $\left.\theta_{W}\right)$ & 70.03 & 2.65 & 2.06 & -0.31 & $-6.32 \times 10^{-2}$ & 2.63 & 3.45 \\
\hline$C E M$ equation & 147.06 & 5.57 & 4.88 & 4.83 & 4.74 & 2.77 & 3.65 \\
\hline JONSWAP & 118.99 & 4.5 & 3.63 & 2.05 & 2.34 & 4.01 & 5.11 \\
\hline
\end{tabular}

series regression, and the BP ANNs have all returned similar correlation patterns, with the complete BP ANN having the highest correlation among them. The effect of the removal of wind direction from the inputs was minimal on the correlation between estimated and target wind speeds. On the other hand, the $C E M$ equation and the JONSWAP model both have presented very different behaviors from the previous techniques, with the tendency to underestimate the wind speeds, and the latter frequently producing spurious peaks.

To quantify these observations, Table 3 shows some measures of accuracy, bias, and precision. Again, the proposed method was the most accurate and precise among the tested techniques, followed by three methods with similar performances. Besides the proposed method, the BP ANNs had the best performance, with the $C E M$ equation and JONSWAP being the least accurate and the most biased toward lower values. Again, the removal of wind direction from the BP ANN input had a small effect on the performance statistics. This enables the use of the BP ANN without wind direction as representative of the method on the gap-filling section, since wind direction is not available within the gaps. While the first five methods in Table 3 have fairly small biases, the proposed method was the only one that had its bias approaching zero if outliers were removed from the analysis. With regard to the precision metrics, the proposed method was the only one to have its standard deviation and interquartile range below $1 \mathrm{~m} \mathrm{~s}^{-1}$, with the four next best performers having standard deviations and interquartile ranges between 2.53 and 3.2 , and 3.36 and 4.36 respectively. The JONSWAP model had the worst precision metric of the three, even if outliers were not considered.

\section{d. Temporal gap filling}

Once the methods are tested, they are applied to the March 2017 dataset, where their results are compared with data from the closest surrounding weather stations, that is, the Red Island Shoal buoy and St. Lawrence weather station. Given that no ground truth data exist within these gaps, precision, accuracy, and bias measurements cannot be retrieved, and considering the distance between the closest neighboring stations, their measurements cannot be considered for ground truth either. However, it can be expected that, on the average, these stations present the same overall variational behavior. Figure 5 presents the linear regression between wind speed data gathered in the mouth of Placentia Bay and the adjacent stations, excluding missing data points. The linear regression analysis shows that both the Red Island Shoal buoy and the St. Lawrence weather station data have high correlation with the wind speed obtained from the mouth of Placentia Bay buoy. Therefore, both datasets can be used as correlation references.

Table 4 presents the correlation coefficients between estimated wind speeds and the ones measured at the same time at the Red Island Shoal buoy $\left(R_{\mathrm{RIS}}\right)$ and the St. Lawrence weather station $\left(R_{\mathrm{StL}}\right)$. With the Red Island Shoal buoy data as reference, the proposed method showed a higher degree of correlation; however, all methods have performed reasonably well. On the other hand, with the St. Lawrence station as reference, the proposed method was slightly surpassed by the SMB equation and the power series regression. However, the methods performed relatively well in all cases, with correlation levels close to the ones obtained by the rest of the dataset.

In light of the analysis performed in the previous section, these results indicate that the proposed method not only has better precision and accuracy than the other methods, but it is also capable of properly filling data gaps with a high degree of correlation with neighboring weather stations.

Figure 6 shows the data collected during the storm at the three stations marked in Fig. 1. From these results it is clear only the proposed method and the JONSWAP model were able to follow the trend of the other two adjacent neighboring stations. All other methods have 


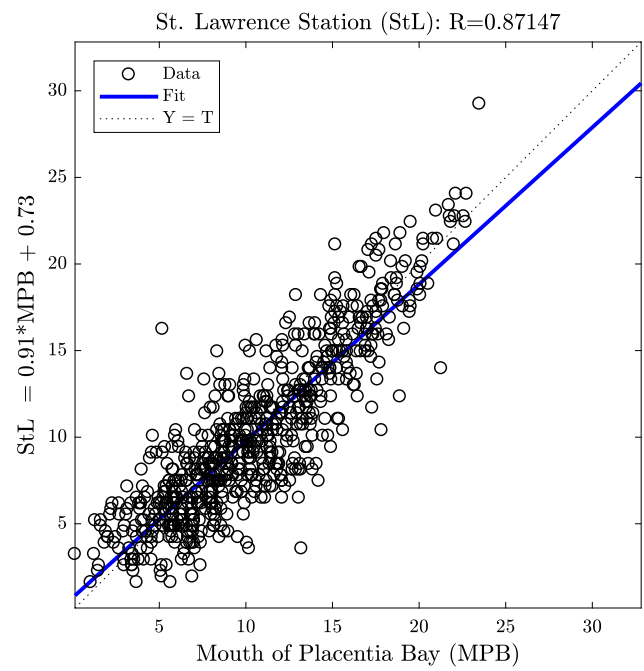

(a)

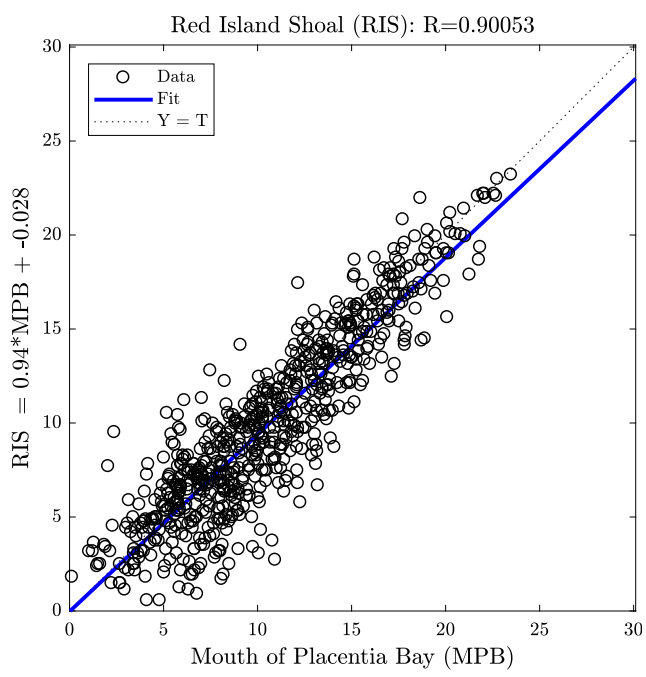

(b)

FIG. 5. Linear regression between measured wind speeds in the mouth of Placentia Bay buoy and (a) St. Lawrence weather station and (b) Red Island Shoal buoy. See Fig. 1 for location reference.

instead returned lower wind speed estimates, contrary to the local trend. However, it is known from the accuracy and bias analysis of the previous section that the JONSWAP model tends to underestimate its predictions, with occasional extreme outliers, while the proposed method consistently presented lower error values. Therefore, by combining both high precision and correlation with the measurements from neighboring weather stations, the proposed method is more suited to produce good wind speed estimates, even during extreme wind events.

\section{Conclusions}

This work presented a wind speed estimation method using a nonlinear autoregressive neural network with exogenous entries. The proposed method was compared with other wind speed estimation techniques, namely, the SMB relationship, power series regression, the BP ANN model, the CEM equation, and the JONSWAP wind speed model. All methods were trained and applied to data obtained by a buoy from SmartAtlantic Alliance, located at the mouth of Placentia Bay, Newfoundland and Labrador, Canada. The goal was to fill gaps in data during the extreme wind events of 11 March 2017.

A cross-correlation analysis was performed in order to select the input data to the proposed method. Among the high cross-correlation measurements, significant wave height was chosen for the input, since it is not a measurement obtained by an anemometer and allows for the expansion of the method for remote sensing applications. All methods requiring training used the same dataset, obtained between May 2013 and February 2014. Then, these methods were tested using a different dataset, collected between March and December 2009.

The proposed method outperformed all other tested wind speed estimation methods during the benchmarking, reaching a correlation coefficient of 0.95 and a mean absolute error of $0.75 \mathrm{~m} \mathrm{~s}^{-1}$. These results are far superior to the second-best method, the backpropagation neural network presented by Zeng et al. (2016).

One of the most marked differences between the proposed method and the other presented estimation techniques is the fact that the proposed model has memory, which allows taking nonconcomitant measurements into account. The choice of a regressive network is supported by the cross-correlation analysis previously mentioned, and the basic theories of wind wave generation and growth (Kinsman 1965). This implies that, however mathematically complicated, there might exist a function that can better represent the relationship between significant wave height and wind

TABLE 4. Correlation between measured and estimated wind speeds within data gaps in the March 2017 dataset.

\begin{tabular}{lll}
\hline \multicolumn{1}{c}{ Method } & $R_{R I S}$ & $R_{S t L}$ \\
\hline Proposed & 0.96 & 0.86 \\
SMB equation & 0.93 & 0.87 \\
Power series & 0.89 & 0.88 \\
BP ANN (no $\theta_{w}$ ) & 0.94 & 0.84 \\
CEM equation & 0.93 & 0.75 \\
JONSWAP & 0.94 & 0.77 \\
\hline
\end{tabular}




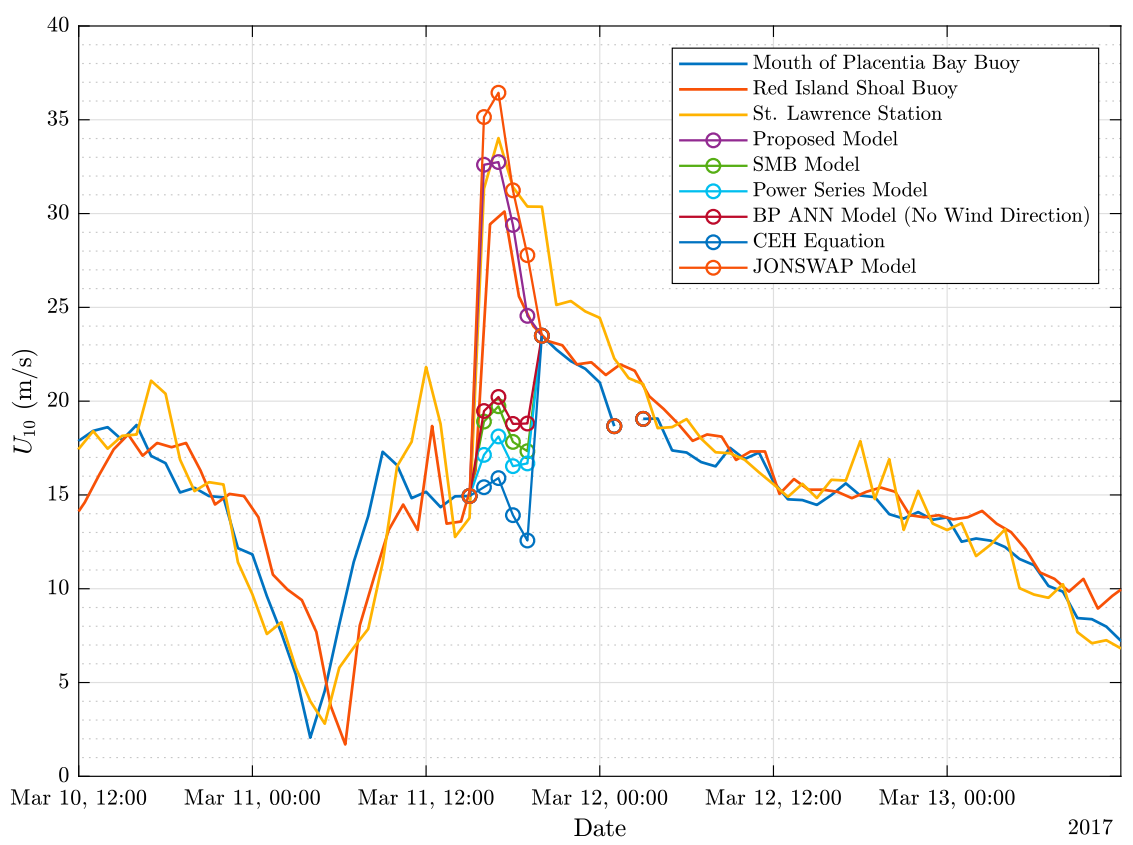

FIG. 6. Comparison between wind speed estimation methods to fill data gaps at the mouth of Placentia Bay during the 11 Mar 2017 storm. Wind speed information from the St. Lawrence weather station and Red Island buoy were presented as references.

speed, and that time delays (or advances) should be considered in this function. Further work can be done on this front in order to prove the importance of memory in empirical wind speed estimation methods.

Future developments of the method might include the application to a remote sensing setting, using HF radar or satellite altimeter data, with both methods being capable of retrieving significant wave height from the ocean surface (Passaro et al. 2015; Gomez et al. 2015). Another possible improvement to the technique is the reduction of the interval between measurements, which can progress to a near-real-time prediction of wind speeds in both buoy and remote sensing settings.

Acknowledgments. The author would like to thank Prof. Cecilia Moloney, PhD., for the early inputs to this work. This work was supported by the Natural Sciences and Engineering Research Council of Canada (NSERC) under Discovery Grants to W. Huang (NSERC RGPIN2017-04508 and RGPAS-2017-507962) and E. W. Gill (NSERC RGPIN-2015-05289).

\section{REFERENCES}

Arthur, R. S., 1951: Wave forecasting and hindcasting. Proceedings of the First Conference on Coastal Engineering, J. W. Johnson, Ed., Coastal Conference Proceedings, Vol. 1, Council on Wave Research, 82-87.

AXYS Technologies, 2017: 3 Metre Buoy. AXYS Technologies Inc., http://axystechnologies.com/products/3-metre-buoy/.
Beale, M. H., M. T. Hagan, and H. B. Demuth, 2017: Neural Network Toolbox: User's Guide. R2018a, The MathWorks Inc., $558 \mathrm{pp}$.

Bretschneider, C. L., 1952: The generation and decay of wind waves in deep water. Eos, Trans. Amer. Geophys. Union, 33, 381-389, https://doi.org/10.1029/TR033i003p00381.

- 1958: Revisions in wave forecasting: Deep and shallow water. Proceedings of Sixth Conference on Coastal Engineering, J. W. Johnson, Ed., Coastal Conference Proceedings, Vo1. 6, 30-67, Council on Wave Research, https://doi.org/10.9753/ icce.v6.3.

Clarizia, M. P., and C. S. Ruf, 2017: Bayesian wind speed estimation conditioned on significant wave height for GNSS-R ocean observations. J. Atmos. Oceanic Technol., 34, 1193-1202, https://doi.org/10.1175/JTECH-D-16-0196.1.

Cybenko, G., 1989: Approximation by superpositions of a sigmoidal function. Math. Control Signals Syst., 2, 303-314, https:// doi.org/10.1007/BF02551274.

Dexter, P. E., 1974: Tests on some programmed numerical wave forecast models. J. Phys. Oceanogr., 4, 635-644, https://doi.org/ 10.1175/1520-0485(1974)004<0635:TOSPNW>2.0.CO;2.

_ , and S. Theodoridis, 1982: Surface wind speed extraction from HF sky wave radar Doppler spectra. Radio Sci., 17, 643-652, https://doi.org/10.1029/RS017i003p00643.

Environment and Climate Change Canada, 2017: Historical data. Government of Canada. Subset used: St. Lawrence Weather Station, March 1-31 2017, accessed 9 November 2017, http:// climate.weather.gc.ca/historical_data/search_historic_data_e.html.

Environment Canada, 2015: MANOBS-Manual of Surface Weather Observations, 7th ed. Amendment 19, Government of Canada, $479 \mathrm{pp}$.

Gaur, S., and M. Deo, 2008: Real-time wave forecasting using genetic programming. Ocean Eng., 35, 1166-1172, https://doi.org/ 10.1016/j.oceaneng.2008.04.007. 
Gomez, R., T. Helzel, L. Wyatt, G. Lopez, D. Conley, N. Thomas, S. Smet, and G. Sicot, 2015: Estimation of wave parameters from $\mathrm{HF}$ radar using different methodologies and compared with wave buoy measurements at the wave hub. OCEANS 2015-Genova, IEEE, 9 pp., https://doi.org/10.1109/OCEANSGenova.2015.7271477.

Hasselmann, K., and Coauthors, 1973: Measurements of windwave growth and swell decay during the Joint North Sea Wave Project (JONSWAP). Ergänzungsheft zur Deutschen Hydrographischen Zeitschrift Reihe A(8) 12, Deutches Hydrographisches Institut, 95 pp., http://resolver.tudelft.nl/uuid: f204e188-13b9-49d8-a6dc-4fb7c20562fc.

Haykin, S. S., 2009: Neural Networks and Learning Machines. 3rd ed. Pearson Education, 936 pp.

Hoffman, D., and O. Karst, 1975: The theory of the Rayleigh distribution and some of its applications. J. Ship Res., 19, 171-191.

Hornik, K., M. Stinchcombe, and H. White, 1989: Multilayer feedforward networks are universal approximators. Neural Networks, 2, 359-366, https://doi.org/10.1016/0893-6080(89)90020-8.

Howden, S., D. Gilhousen, N. Guinasso, J. Walpert, M. Sturgeon, and L. Bender, 2008: Hurricane Katrina winds measured by a buoy-mounted sonic anemometer. J. Atmos. Oceanic Technol., 25, 607-616, https://doi.org/10.1175/2007JTECHO518.1.

Huang, W., S. Wu, E. Gill, B. Wen, and J. Hou, 2002: HF radar wave and wind measurement over the eastern China Sea. IEEE Trans. Geosci. Remote Sens., 40, 1950-1955, https://doi.org/ 10.1109/TGRS.2002.803718.

Kinsman, B., 1965: Wind Waves: Their Generation and Propagation on the Ocean Surface. Courier Corporation, 676 pp.

Kitaigorodskii, S. A., 1962: Applications of the theory of similarity to the analysis of wind-generated wave motion as a stochastic process. Izv. Akad. Nauk SSSR, Ser. Geofiz., 1, 105-117.

Li, L., X. Wu, X. Xu, and B. Liu, 2012: An empirical model for wind speed inversion by HFSWR. Wuhan Daxue Xuebao Xinxi Kexueban, 37, 1096-1099, http://ch.whu.edu.cn/EN/abstract/ article_314.shtml.

Li, N., M. Wei, X. Mu, and C. Zhao, 2015: A support vector machine-based VVP wind retrieval method. Atmos. Sci. Lett., 16, 331-337, https://doi.org/10.1002/asl2.564.

,,-- Y. Yu, and W. Zhang, 2017: Evaluation of a support vector machine-based single-Doppler wind retrieval algorithm. J. Atmos. Oceanic Technol., 34, 1749-1761, https://doi.org/ 10.1175/JTECH-D-16-0199.1.

Londhe, S., S. Shah, P. Dixit, T. B. Nair, P. Sirisha, and R. Jain, 2016: A coupled numerical and artificial neural network model for improving location specific wave forecast. Appl. Ocean Res., 59, 483-491, https://doi.org/10.1016/j.apor.2016.07.004.

MacEachern, D., 2017: Hurricane-strength winds tear swath of destruction across northeast Avalon. CBC/Radio Canada, http://www.cbc.ca/news/canada/newfoundland-labrador/winterweather-returns-1.4020934.

Massel, S. R., 2013: Ocean Surface Waves: Their Physics and Prediction. Advanced Series on Ocean Engineering, Vol. 36, World Scientific, 667 pp.
NDBC, 2018: National Data Buoy Center, http://www.ndbc.noaa.gov.

Nikoo, M. R., and R. Kerachian, 2017: Wave height prediction using artificial immune recognition systems (AIRS) and some other data mining techniques. Iran. J. Sci. Technol. Trans. Civ. Eng., 41, 329-344, https://doi.org/10.1007/s40996017-0067-y.

Passaro, M., L. Fenoglio-Marc, and P. Cipollini, 2015: Validation of significant wave height from improved satellite altimetry in the German bight. IEEE Trans. Geosci. Remote Sens., 53, 2146-2156, https://doi.org/10.1109/TGRS.2014.2356331.

Pierson, W. J., and L. Moskowitz, 1963: A proposed spectral form for fully developed wind seas based on the similarity theory of S. A. Kitaigorodskii. New York University Geophysical Sciences Laboratory Rep., 26 pp.

Powell, M. D., 1982: The transition of the Hurricane Frederic boundary-layer wind field from the open Gulf of Mexico to landfall. Mon. Wea. Rev., 110, 1912-1932, https://doi.org/ 10.1175/1520-0493(1982)110<1912:TTOTHF $>2.0 . C O ; 2$.

Resio, D. T., and C. L. Vincent, 1977: Estimation of winds over the Great Lakes. J. Waterw. Port Coastal Ocean Div., 103, 265-283.

Siegelmann, H. T., B. G. Horne, and C. L. Giles, 1997: Computational capabilities of recurrent NARX neural networks. IEEE Trans. Syst. Man Cybern., 27B, 208-215, https://doi.org/10.1109/ 3477.558801.

SmartAtlantic Alliance, 2017: SmartBay-Placentia Bay, Newfoundland. SmartAtlantic Alliance, http://www.smartatlantic. ca/PlacentiaBay/.

Snoddon, R., 2017: A storm we won't soon forget. CBC/Radio Canada, http://live.cbc.ca/Event/Ryan_Snoddon/768237073.

Sontag, E. D., 1992: Feedback stabilization using two-hidden-layer nets. IEEE Trans. Neural Networks, 3, 981-990, https://doi.org/ 10.1109/72.165599.

Stewart, R. H., 2008: Introduction to Physical Oceanography. Stewart, $345 \mathrm{pp}$.

Suckling, E. B., G. J. van Oldenborgh, J. M. Eden, and E. Hawkins, 2017: An empirical model for probabilistic decadal prediction: Global attribution and regional hindcasts. Climate Dyn., 48, 3115-3138, https://doi.org/10.1007/ s00382-016-3255-8.

Tokinaga, H., and S.-P. Xie, 2011: Wave- and anemometerbased sea surface wind (WASwind) for climate change analysis. J. Climate, 24, 267-285, https://doi.org/10.1175/ 2010JCLI3789.1.

U.S. Army Corps of Engineers, 2012a: Coastal Engineering Manual: Part II; Coastal Hydrodynamics. EM 1110-2-1100, Books Express Publishing, $624 \mathrm{pp}$.

— 2012b: Coastal Engineering Manual: Part III; Coastal Sediment Processes. EM 1110-2-1100, Books Express Publishing, $480 \mathrm{pp}$.

Zeng, Y., H. Zhou, H. Roarty, and B. Wen, 2016: Wind speed inversion in high frequency radar based on neural network. Int. J. Antennas Propag., 2016, 2706521, https://doi.org/10.1155/ 2016/2706521. 